\title{
Some stages of information processing in a choice reaction-time task*
}

\author{
SANDRA R. PALEF† \\ University of Toronto, Toronto 181, Ontario, Canada
}

\begin{abstract}
The purpose of the present experiment was to demonstrate the serial nature of information processing by revealing stages which intervene between a stimulus and a choice response. Three variables were manipulated in a choice reaction-time task involving 12 female Ss: stimulus quality, type of match between test stimulus and alternatives, and number of alternative stimuli. All three variables had significant effects on mean reaction time. The only significant interaction was between type of match and number of alternatives, and this was small in magnitude. The results suggested the existence of at least two additive stages of processing, one affected by stimulus quality and one affected by both type of match and number of alternatives.
\end{abstract}

One of the assumptions on which theories of human information processing are based is the idea that the time from the onset of a stimulus to the occurrence of a response can be divided into a series of stages, each representing a different operation. Some theories (Sternberg, 1969a) assume that the stages are additive, i.e., they do not overlap in time, while others assume distinct stages but not the additivity of stages (Moray, 1967; Taylor, Lindsay, \& Forbes, 1967). The additive-factor method (Sternberg, 1969a, b) provides a test of the notion of additivity by assuming that if stage durations are additive, then variables which affect those stages separately would have additive effects on reaction time. Furthermore, variables which affect the same stage would have interacting effects on reaction time. As long as the variables manipulated are expected to influence separate stages and are not confounded in the experimental task employed, then the finding of additivity between variables suggests corresponding additive stages, and interacting effects suggest a stage influenced by both variables.

The purpose of the present experiment was to test the notion of additivity of stage durations in a choice reaction-time task. It was assumed that in order to do this task, the $S$ would have to perform at least the following three operations: encode or identify the test stimulus, match the test stimulus identification to the stimulus alternatives, and select an appropriate response. In order to discover whether these three processes represent additive stages of information processing, the following three variables, which are known to affect reaction time, were experimentally manipulated: stimulus quality, type of stimulus match, and number of alternative stimuli. Evidence exists in the literature that

*This article is based on some of the research submitted in partial fulfillment of the requirements for the MA degree. Carleton University.

$\div$ Requests for reprints should be sent to Sandra R. Palef, Department of Psychology. University of Toronto. Toronto 181. Ontario. Canada. these variables affect particular processes. Reaction time to intact stimuli is faster than reaction time to degraded stimuli (Sternberg, 1967), presumably because the encoding process is made more time-consuming by the degradation. Hence, this variable would likely affect the first operation mentioned above. Matching physically identical stimuli requires less time than matching stimuli on the basis of name identity (Posner, 1969), presumably because more time is required to identify the name of the stimulus than to identify it in physical terms. Finally, the increase in reaction time with increases in the number of alternative stimuli probably results from an increase in the time to select an appropriate response. Based on these explanations, it was predicted that by simultaneously manipulating these three variables in a choice reaction-time situation, three additive stages of processing would be revealed. The test stimulus would likely be encoded before any matching took place and certainly before response selection. Matching would probably be completed before response selection began. Therefore, all two-way interactions, as well as the three-way interaction, would be nonsignificant.

In general, the finding of one or more instances of additivity would be support for the notion that information processing in a choice reaction-time task involves operations which are arranged in series. An interaction of factors would have implications about the functions of the stages revealed in terms of the combination of variables affecting them.

\section{METHOD}

\section{Subjects}

Twelve undergraduate females from Carleton University served as Ss. They were paid $\$ 1.50$ an hour for their services.

\section{Apparatus and Stimuli}

A Leitz-Pradovit slide projector permitted the presentation of slides on a screen attached to the top of the response unit. The 
Table 1

Analysis of Variance for Mean Reaction Time Scores for Conditions Case of Letters Memorized, Number of Alternatives, Type of Match, and Stimulus Quality

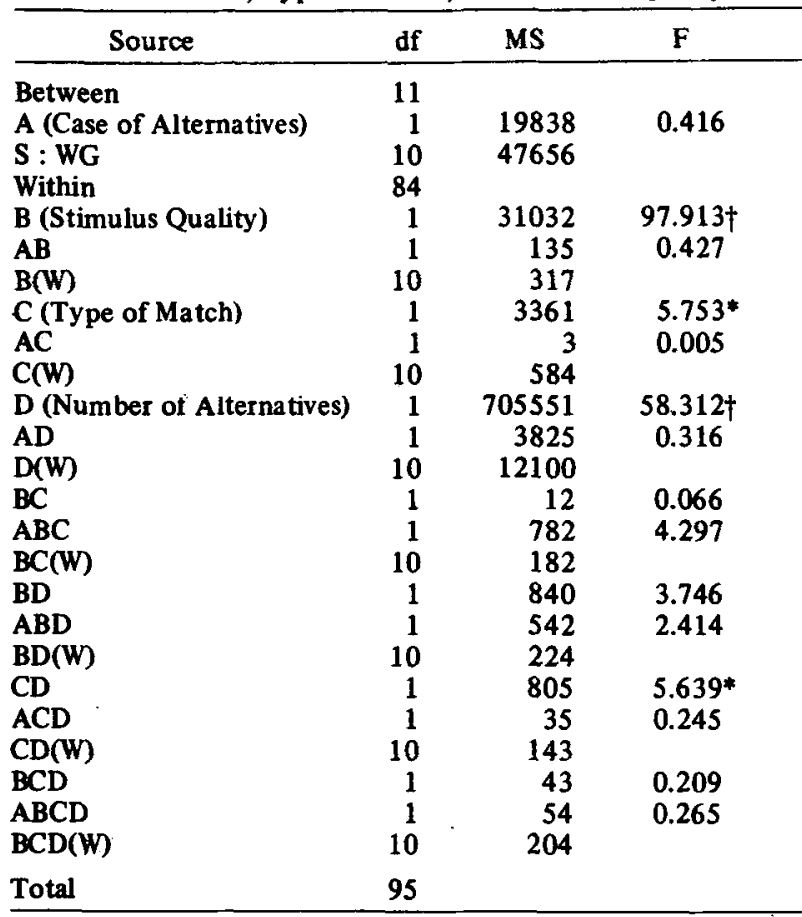

$p<.05 \quad t_{p}<.001$

response keys consisted of four push buttons, two for the left hand and two for the right hand, mounted on the response box. In the two-choice condition, only the two center buttons were used, whereas in the four-choice condition, all buttons were used. Reaction time in milliseconds was measured from the onset of the stimulus display to the S's response by means of a millisecond timer. The timer controlled the duration of the warning tone and the interval between the onset of the warning tone and the onset of the stimulus display. A panel of lights, each illuminated when a given response key was pressed, permitted $E$ to check the accuracy of responses.

The stimuli consisted of the letters $R, H, M, F, B$, and D. Four of the letters were assigned randomly as stimulus alternatives in the four-choice condition for a given $S$, while the remaining two letters were assigned to the two-choice condition. The one restriction was that the letters $B$ and $D$ could not be assigned to the same set, since pilot studies indicated some confusion in discriminating between these letters in a choice situation. Each letter was made into four $35-\mathrm{mm}$ slides, one for each combination of case of letter (upper and lower) and quality (intact and degraded). To degrade the letters, a black and white checkerborad overlay was placed on top of the letter before it was photographed. The appropriate stimulus alternatives for each $S$ were arranged in a horizontal line on the response unit right under the screen. Each of these stimuli corresponded to a particular response button.

\section{Procedure}

Each $S$ was tested individually. The $E$ emphasized the existence of the stimulus alternatives on the response unit and instructed $S$ to press an appropriate button as rapidly as possible without making mistakes, for each presentation of one of the alternatives, no matter what the case or the quality. The $\mathrm{S}$ was encouraged to use the visually displayed alternatives in making her decision. Each trial consisted of a warning tone of $75 \mathrm{msec}$ duration, followed, after an interval of $500 \mathrm{msec}$, by the test stimulus, which was visible until $S$ responded, and then by an intertrial interval varying around $4 \mathrm{sec}$. This corresponds to the time the $E$ required to reset the accuracy indicator, record reaction time, reset the timer, and press the start button.

\section{Design}

A 2 by 2 by 2 by 2 factorial design was employed with all Ss receiving both a two-choice and a four-choice set of alternatives, with physical and name matches in each set, and intact and degraded stimuli in each set. Half the Ss were presented with uppercase letters as alternatives and half with lowercase letters to prevent a possible confounding of the type of match variable by case of alternatives. However, the effect of this variable is not of inieresi here, and does not enter into the theoretical argument. Each $S$ participated in three 1-h sessions, separated by approximately $24 \mathrm{~h}$. The first session was considered practice. Each session was divided into two parts, one for the two-choice condition and one for the four-choice condition, with order of conditions counterbalanced across Ss and experimental sessions. Intact and degraded stimuli occurred equally often in each part, upper- and lowercase letters occurred equally often, and each of the two or four possible stimulus letters occurred equally often. A different random ordering of each of these variables was used for each $S$ on each day.

Excluding practice trials at the beginning of each part and following breaks, the procedure yielded a total of 336 trials for each $S$ on each of the 2 days which were subject to experimental analysis, or a total of 84 trials per condition for each $S$ over the 2 experimental days.

\section{RESULTS}

Since the interest in this experiment was one of errorless performance, incorrect responses were not included in the analyses. Relative frequency of errors was minimal, with an average error rate of about $1 \%$ for experimental trials. Mean reaction time scores based on correct responses were determined for each $S$ for each condition over the two experimental sessions. The data were analyzed, using a four-way analysis of variance with case of alternatives (upper vs lower), number of alternatives (two vs four), type of match (physical vs name), and stimulus quality (intact vs degraded) as the variables. The last three mentioned variables involved repeated measures. A summary of the analysis is presented in Table 1. Since the analysis indicated no significant main effect for case of alternatives and no significant interactions involving case of alternatives, the data were collapsed across this variable. The resulting means are shown graphically in Fig. 1, which presents mean reaction time as a function of number of alternatives for the physical and name match conditions. Each point in the figure is a mean reaction time based on the data for all 12 Ss. The analysis showed significant main effects for number of alternatives $[F(1,10)=$ $58.31, p<.001]$, type of match $[F(1,10)=5.75$, $\mathrm{p}<.05]$, and stimulus quality $[\mathrm{F}(1,10)=97.91$, $\mathrm{p}<.001]$. As shown in Fig. 1, reaction time was greater for the four-choice condition than for the two-choice condition, reaction time was greater for name matches than for physical matches, and reaction time was greater 
Fig. 1. Reaction time as a function of number of alternatives, for physical and name matches and intact and degraded stimuli.

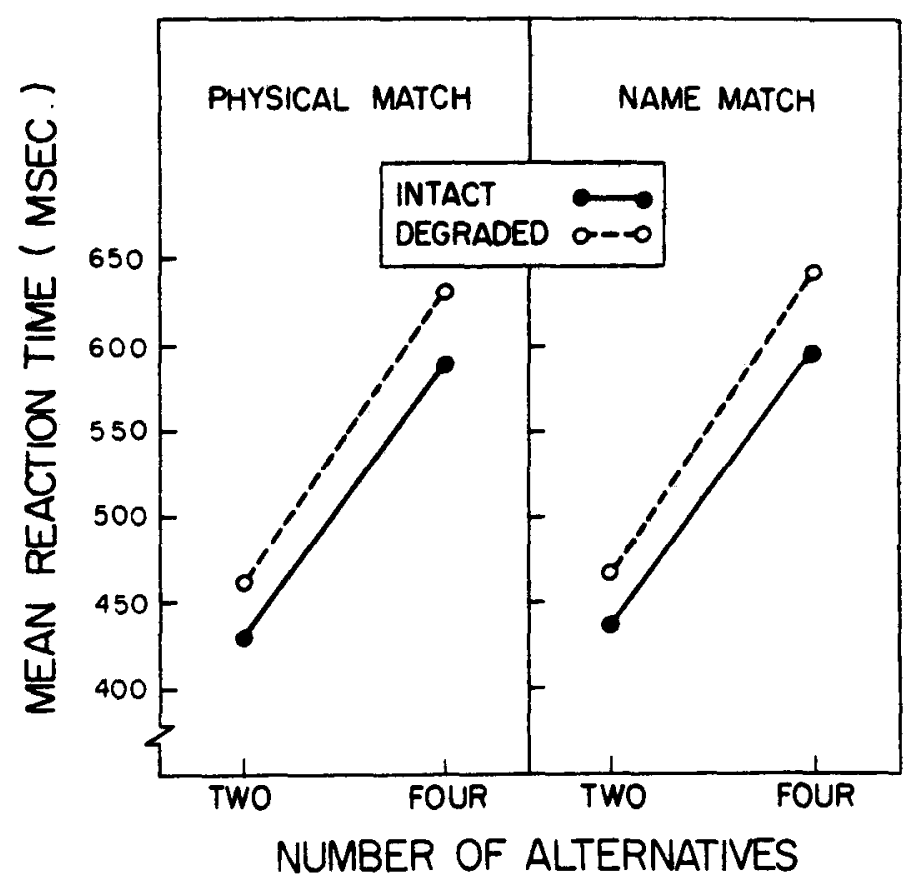

for degraded stimuli than for intact stimuli. The only significant interaction was between type of match and number of alternatives $[F(1.10)=5.64, p<.05]$. Physical matches were, on average, $6 \mathrm{msec}$ faster than name matches in the two-choice condition and $18 \mathrm{msec}$ faster in the four-choice condition. An examination of this significant interaction using Sandler's (1955) A statistic indicated that physical matches were significantly faster than name matches only in the four-choice condition $(A=.20, p<.05)$. However, the magnitude of the interaction was very small compared to the main effects-the mean square for the interaction was 805 compared to 3.361 for type of match and 705.551 for number of alternatives. None of the other interactions was significant. Although the difference in reaction time between intact and degraded stimuli was greater for four alternatives than for two (43 $\mathrm{msec}$ compared to $30 \mathrm{msec}$ ), this interaction was not significant. Evidently, the higher degree of variability associated with this interaction as compared to the Match by Alternatives interaction was responsible for the significance of the latter and not the former. The experiment was designed to provide a reasonably sensitive test of the interactions involved. Since the Quality by Alternatives interaction did not prove to be significant, appropriate conclusions must be drawn.

\section{DISCUSSION}

The results of the present experiment offer some evidence for the notion that information processing in a choice reaction-time task involves a series of distinct operations which to not overlap in time. The finding of an additive relationship between stimulus quality and type of match, and between stimulus quality and number of alternatives. suggests that the choice reaction-time task of pressing buttons to visually presented letters involves at least two separate stages of processing. One stage is affected by stimulus quality, while the other stage is affected by number of alternatives. The finding that type of match and stimulus quality did not interact significantly, whereas type of match and number of alternatives did interact, suggests that type of match affects the same stage as does number of alternatives and that type of match does not affect the stage that is influenced by stimulus quality.

Assuming that the variables manipulated in this experiment actually affect the operations described above, i.e., that quality affects encoding, type of match affects a comparison or matching operation, and number of alternatives affects response selection, then one can draw further conclusions from the results. The finding that type of match did not interact with stimulus quality suggests that the form of the test stimulus that is compared with the alternatives is not a raw image. but is abstracted enough so that degradation does not affect the matching. The finding that stimulus quality did not interact with number of alternatives suggests that encoding and response selection are completely separate in time. The interaction between type of match and number of alternatives might suggest that the time to match the test stimulus identification with the alternative stimuli overlaps with the time to select a response. On the other hand, it is not unreasonable to suggest that number of alternatives would affect the matching process as well as response selection. If this were the case. then one would expect an interaction between type of match and number of alternatives which was at least the magnitude of the one found here. This experiment points to the conclusion that a choice 
reaction-time task does display the additivity of stage durations which is assumed by theories of human information processing. The study demonstrates the usefulness of the additive-factor approach in a choice reaction-time task.

\section{REFERENCES}

Moray, N. Where is capacity limited? A survey and a model. Acta Psychologica, 1967, 27, 84-92.

Posner, M. I. Abstraction and the process of recognition. In G. Bower and J. T. Spence (Eds.), Psychology of learning and motivation. Vol. 3. New York: Academic Press, 1969.

Sandler, J. A test of the significance of the difference between the means of correlated measures, based on a simplification of the Student's t. British Journal of Psychology, 1955, 46, 225-226.

Stemberg, S. Two operations in character recognition: Some evidence from reaction-time measurements. Perception \& Psychophysics, 1967, 2, 45-53.

Sternberg, S. The discovery of processing stages: Extensions of Donders' method. Acta Psychologica, 1969a, 30, 276-315.

Sternberg, S. Memory-scanning: Mental processes revealed by reaction-time experiments. American Scientist, 1969b, 57, 421-457.

Taylor, M. M., Lindsay, P. H., \& Forbes, S. M. Quantification of shared capacity processing in auditory and visual discrimination. Acta Psychologica, 1967, 27, 223-229.

(Received for publication February 11, 1972; revision received July $12,1972$. ) 